\title{
Evaluation of the thin-skin approximation boundary element method for electromagnetic induction scattering problems
}

DOI:

10.1109/SAS.2016.7479843

Link to publication record in Manchester Research Explorer

Citation for published version (APA):

O'Toole, M., Marsh, L., Peyton, A., Yin, W., \& Davidson, J. (2016). Evaluation of the thin-skin approximation boundary element method for electromagnetic induction scattering problems. In IEEE Sensors Applications Symposium (SAS), 2016 IEEE. https://doi.org/10.1109/SAS.2016.7479843

Published in:

IEEE Sensors Applications Symposium (SAS), 2016

\section{Citing this paper}

Please note that where the full-text provided on Manchester Research Explorer is the Author Accepted Manuscript or Proof version this may differ from the final Published version. If citing, it is advised that you check and use the publisher's definitive version.

\section{General rights}

Copyright and moral rights for the publications made accessible in the Research Explorer are retained by the authors and/or other copyright owners and it is a condition of accessing publications that users recognise and abide by the legal requirements associated with these rights.

\section{Takedown policy}

If you believe that this document breaches copyright please refer to the University of Manchester's Takedown Procedures [http://man.ac.uk/04Y6Bo] or contact uml.scholarlycommunications@manchester.ac.uk providing relevant details, so we can investigate your claim.

\section{OPEN ACCESS}




\title{
Evaluation of the Thin-Skin Approximation Boundary Element Method for Electromagnetic Induction Scattering Problems
}

\author{
M. D. O'Toole, J. L. Davidson, L. A. Marsh, W. Yin and A. J. Peyton \\ School of Electrical and Electronic Engineering \\ The University of Manchester \\ Manchester, M13 9PL, UK \\ Email: michael.otoole@manchester.ac.uk
}

\begin{abstract}
A conductive object subject to an applied varying magnetic field will emit a secondary magnetic field or scattered field. The scattered field is dependent on the geometry of the object and its material properties (conductivity, permeability, etc). If we can calculate the scattered field for a given geometry (the scattering problem), we can infer the material properties from the detected electromagnetic response. Our motivation is the production of induction based classifiers for object and material classification. Applications include sorting of high value scrap metal and identifying UXO from clutter in landmine clearance. To this end, we require methods of solving the scattering problem quickly and accurately.

In this paper, we evaluate the thin-skin approximation boundary element method. The method offers a particularly compact formulation of the scattering problem which is quick to solve. We compare this method to the more established finite element method. We find that larger objects at higher frequencies and conductivities appear to give good agreement between the two methods. However, the agreement breaks down for smaller objects even when the frequency or conductivity is relatively high for typical induction based sensing. This is especially true when the object has a complex geometry. This imposes limitations on the practical usefulness of this approach.
\end{abstract}

\section{INTRODUCTION}

The boundary element method, or method of moments, is a well-established procedure for solving electromagnetic scattering problems [1]. The method uses a discretisation of the surface of the scattering object into a series of source points, observation points and linear basis functions in order to pose the scattering problem in the form of a linear matrix function. We can then solve for the unknown source points by using classical matrix inversion techniques to obtain the magnetic field across the entire surface of the object. With the object boundary conditions found, we can then solve for the magnetic field at any point by using the appropriate Green's function.

The boundary element method is particularly advantageous where we wish to avoid discretising an entire set of domains in to a volumetric mesh, for instance when using finite element or finite time difference methods. We need only discretise the surface or boundary using planar meshes such as triangular meshes. In some cases, such as when we only need to find magnetic field in a small number of locations, this method can offer significant solution-time improvements over alternative solvers.

In the present work, we are motivated by two applications for which the boundary element method offers a promising solution. The first is in the estimation of the magnetic polarizability tensor of metal objects [2]. The magnetic polarizability tensor is a function of the dimensions, shape, material composition, and orientation of a metal object. If we know the tensor of the object beforehand, we can use it to distinguish different metallic objects from one another purely from their magnetic response. This approach has significant application in areas such as walk-through metal detectors [3], [4] and in landmine detection and clearance [5].

A boundary element approach to predicting the magnetic polarizability tensor has been previously reported by Lu et al. [2]. However, this only addressed the asymptotic case of a perfect electrical conductor. We follow this work by extending to finite but high conductivities.

The second application is identifying metals in scrap metal sorting. Current processes such as permanent magnets and eddy-current separation can sort large proportions of the metal samples, however, they leave a significant fraction of valuable metals and alloys such as brass, bronze, aluminium and copper [6]. Assuming the shape of the samples are known, we can use a fast solver to obtain the scattering of these objects in response to an excitation and thus obtain a prediction of their conductivity and material type.

In this paper, we evaluate a boundary element method that uses a thin-skin approximation to produce a particularly compact formulation of the scattering problem for high conductive objects where skin-depth is small relative to the characteristic dimensions of object [7], [8]. The compactness of the formulation means that the resultant matrix problem is small and the consequent inversion time to solve for the boundary elements is kept short. We examine the accuracy of the field solutions using this method by comparing with established finite-element methods using a small range of simulated test objects. 


\section{THIN-SKIN APPROXIMATION BOUNDARY ELEMENT METHOD}

In this section, we provide only a brief overview of the method formulated by Sun et al. [7], [8]. We refer the interested reader to these original references for a more detailed and thorough explanation of this approach.

Consider a simply connected conductive object with surface $S$ existing in an external space that has magnetic permeability at or close to free space and a conductivity close to zero. We subject the object to an excitation magnetic field which in turn induces a secondary magnetic field in the external region. By further assuming that the magnetic field in the external region is quasi-static, we can state that the magnetic field in the external region is irrotational and can therefore be described in terms of magnetic scalar potentials $\psi^{e}$ and $\psi^{p r}$, where the first term is the (overall) potential of the field in the exterior region and the second denotes the potential due to the excitation field.

The following integral equation describes the magnetic scalar potential at an observation point $\mathbf{r}$ in the external region or on the surface of the object,

$$
\begin{aligned}
\psi^{p r}(\mathbf{r}) & =\beta(\mathbf{r}) \psi^{e}(\mathbf{r}) \\
& +\int_{S}\left(\psi^{e}\left(\mathbf{r}^{\prime}\right) \frac{\partial g\left(\mathbf{r}, \mathbf{r}^{\prime}\right)}{\partial n^{\prime}}+g\left(\mathbf{r}, \mathbf{r}^{\prime}\right) H_{n}^{e}\left(\mathbf{r}^{\prime}\right)\right) d S^{\prime}
\end{aligned}
$$

where $g\left(\mathbf{r}, \mathbf{r}^{\prime}\right)=1 /\left(4 \pi\left|\mathbf{r}-\mathbf{r}^{\prime}\right|\right)$ is the Green's function for the Laplace equation, $\mathbf{r}^{\prime}$ are source points on the surface of the object, $n$ is a surface normal with positive pointing outwards and $H_{n}^{e}$ the component of the magnetic field normal to the surface. The term $\beta(\mathbf{r})$ equals the exterior solid angle required to exclude the singularity, divided by $4 \pi$, such that $\beta=1$ when $\mathbf{r}$ is in the external region, $\beta=0$ when $\mathbf{r}$ is inside the object and $\beta=0.5$ when $\mathbf{r}$ is on a perfectly flat region of the object's surface. In the present work, this is calculated by $\beta=1-\Omega / 4 \pi$, where $\Omega$ is the solid angle at $\mathbf{r}$ subtended by the surface of the object.

The thin-skin approximation assumes that the following relation applies to the surface of the object,

$$
\frac{\partial H_{n}}{\partial n}=-i \alpha_{n} k H_{n}(\mathbf{r}), \quad \mathbf{r} \in S
$$

where $H_{n}$ is the component of the magnetic field normal to the object surface, $i=\sqrt{-1}$, and $k=\sqrt{\sigma \omega \mu / i}\left(\mathrm{~m}^{-1}\right)$ with $\omega$ the frequency $(\mathrm{rad} / \mathrm{sec}), \sigma$ the conductivity $(\mathrm{S} / \mathrm{m})$ and $\mu$ the magnetic permeability $(\mathrm{H} / \mathrm{m})$. The term $\alpha_{n}$ is a correction factor which accounts for deviation from the ideal case, the ideal case being when the field inside the object is governed $H_{n}(n)=H_{n}(0) e^{-i k n}$ where $n$ is the distance from the surface. In what follows we assume the ideal case is true and thus $\alpha_{n}=1$.

We now wish to pose equations (1) and (2) in the form of a boundary element problem so that we can solve for surface $\psi_{e}$ and $H_{n}$ and then for the same terms in the exterior region. First, the surface of the shape is approximated by a triangular mesh consisting of $N$ node points. We then introduce an approximation for $\psi_{e}$ and $H_{n}^{e}$ on the surface,

$$
\psi^{e}(\mathbf{r})=\sum_{j}^{N} \psi_{j}^{e} \varphi_{j}(\mathbf{r}), \quad H_{n}^{e}(\mathbf{r})=\sum_{j}^{N} H_{n, j}^{e} \varphi_{j}(\mathbf{r})
$$

where $\psi_{j}^{e}$ and $H_{n, j}^{e}$ are respectively the scalar potential and normal magnetic field at node $j$ and $\varphi_{j}(\mathbf{r})$ is a linear basis function which is equal to one one when $\mathbf{r}$ corresponds to the $j^{\text {th }}$ node point and declines to zero as $\mathbf{r}$ approaches adjacent nodes.

The boundary element problem is derived using equations (1) and (2), the approximations in (3), and the divergence relation $\nabla \cdot \mathbf{H}=0$ in the object interior (see Sun et al. [7] for the detailed derivation). For the $i^{t h}$ observation point,

$$
\begin{array}{r}
\psi^{p r}\left(\mathbf{r}_{\mathbf{i}}\right)=\sum_{j=1}^{N}\left(\beta\left(\mathbf{r}_{\mathbf{i}}\right) \psi_{i}^{e}+\psi_{j} \int_{S} \varphi_{j}\left(\mathbf{r}^{\prime}\right) \frac{\partial g\left(\mathbf{r}_{i}, \mathbf{r}^{\prime}\right)}{\partial n^{\prime}} d S^{\prime}\right. \\
\left.+H_{n, j}^{e} \int_{S} g\left(\mathbf{r}_{i}, \mathbf{r}^{\prime}\right) \varphi_{j}\left(\mathbf{r}^{\prime}\right) d S^{\prime}\right) \\
0=\sum_{j=1}^{N}\left(\psi_{j}^{e} \int_{A_{i}} \nabla \varphi_{i} \cdot \nabla \varphi_{j} d A\right. \\
\left.+\frac{H_{n, j}^{e}}{\mu_{r}}\left[\frac{1}{A_{i}}-i k \alpha_{n}\right] \int_{A_{i}} \varphi_{i} \varphi_{j} d A\right)
\end{array}
$$

where $A_{i}$ is the area of the surface triangular elements around node $i$. We can solve for the unknown boundary terms $\psi_{1}^{e}, \psi_{2}^{e}, \ldots, \psi_{N}^{e}$ and $H_{n, 1}^{e}, H_{n, 2}^{e}, \ldots H_{n, N}^{e}$ by choosing observation points coincident with the nodes. This gives us sufficient equations $(2 N)$ to solve for each unknown via any standard matrix inversion approach. We can then use the boundary terms to calculate the magnetic scalar potential, and therefore the magnetic field, for any observation point outside of the object.

\section{METHOD}

We evaluate the TSA-BEM by comparing the resultant scattered field computed by this method for four distinct high conductivity $\left(10^{7} \mathrm{~S} / \mathrm{m}\right)$ objects with the scattered field computed by a commercial finite element solver for the same objects and same excitation field. For the present work, the excitation is equivalent to a cylindrical coil of $10 \mathrm{~cm}$ in radius and $100 \mathrm{~cm}$ in length with central axis coincident with the $\mathrm{x}$ axis, providing a nearly uniform central field of approximately $1 \mathrm{~A} / \mathrm{m}$.

Four test objects are used with different geometries and sizes: A cylinder, an ellipsoid, a right angle or L-shape, and $\mathrm{C}$-shape or cut ring. The dimensions of each object are shown in figure 1 . The cylinder is chosen to follow recent work on using the perfect electrical conductor version of this boundary element method [2], i.e. when $H_{n}^{e}$ in equation (2) is zero. The dimensions and mesh from the cylinder in this work are kept the same for the present work. The ellipsoid is selected to follow the original work which proposed the TSA approach [7]. This work evaluated the method for spheres, ellipsoids 


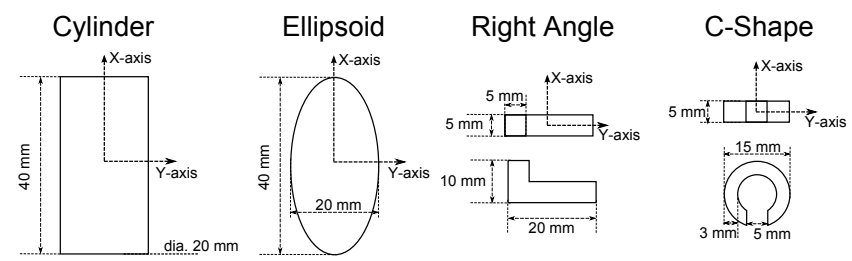

Fig. 1. Geometry of the test objects.

and deformed ellipsoids. The right-angle and $\mathrm{C}$-shape were selected from our test library of real components for their interesting geometry.

\section{A. Implementation of the thin skin approx. boundary element method}

The thin-skin approximation boundary element method was implemented in Matlab 2014b (Mathsworks inc, USA) and used surface triangular meshes generated by Netgen 5.3 [9] from CAD drawn STL files created for each object. The number of nodes and surface triangles for each object are as follows:

- Cylinder - nodes 146, triangles 288,

- Ellipsoid - nodes 336, triangles 668,

- Right angle - nodes 274, triangles 544,

- C-shape - nodes 723, triangles 1442.

The number of surface triangles are deliberately kept small as we are investigating this method primarily for its ability to produce rapid results to scattering problems in high throughput applications. Large numbers of surface triangles increase the time to produce the mesh and increase the size of the required matrix inversion, which is in general of $N^{3}$ complexity. There are a number of methods, such as the fast multi-pole method and precorrected FFT [10], which can improve the inversion time. However, these methods necessarily incur some overhead costs in computation. Investigation of these methods falls out of the scope of our current work.

The equations (4) and (5) require integration of the Laplacian Green's function and it's normal derivative over the surface triangles. This introduces singularities when the node and observation point coincide. We resolve the integrals analytically using the method proposed by Graglia [11] for integrating 3-D Green's functions over planar triangles.

\section{B. Finite element method}

Simulations were performed using the commercial Finite Element Method solver, Ansys Maxwell v16. Each test object was positioned within the central region of the coil and simulations were performed for a total of 31 frequencies from $10 \mathrm{~Hz}$ to $10 \mathrm{MHz}$ with 5 logarithmic based increments per decade frequency change. Typical total meshing levels was in the order of $100 \mathrm{k}$ tetrahedral elements and solution times for a single object at all frequencies was in the order of $0.5 \mathrm{hrs}$ running on Intel Core i7-2600 (3.4 GHz).

\section{RESUlTS AND DisCUSSION}

We compare the scattered field computed by the boundary element with the field computed by the finite element method. By scattered field, we refer to the magnetic field solely generated by the object in consequence to the excitation. In other words, the field in the external region with the initial excitation subtracted. Field points are calculated across the exterior region of the object at a resolution of $4 \mathrm{~mm}$.

Field vector data exported from the finite element method solver was visualized with ParaView [12] software. The associated secondary-field vector cloud for each test object was visualized at all frequencies using the streamtracer and glyph filters of ParaView in order to give a 3D representation of the form and direction of the calculated fields. This was performed for both boundary element and finite element data in order to give a direct and accurate 3D visual comparison between the two methodologies. Examples at $10 \mathrm{kHz}$ and 1 $\mathrm{MHz}$ are shown in figure 2 for objects with conductivity of $\sigma=3.5 \times 10^{7} \mathrm{~S} / \mathrm{m}$. In this figure, the real component of the boundary element computed magnetic field is shown in the form of streamlines across a plane. The finite element computed values are shown for $1 \mathrm{MHz}$ with red glyph arrows superimposed over the streamlines. These images have also been rendered in to animations showing finite and boundary element data over different frequencies ${ }^{1}$.

Figures 3 and 4 show the real and imaginary $\mathrm{x}$-axis component of the scattered field $\left(H_{x}^{s}\right)$ in a localised region below the object. We define the bottom of the object as being the face looking towards the negative $x$-axis. Figure 3 shows the results over a range of wavenumbers at a single observation point in space at $\mathrm{y}=2 \mathrm{~mm}, \mathrm{z}=2 \mathrm{~mm}$ and $\mathrm{x}=8 \mathrm{~mm}$ below the bottom surface. It is feasible to conceive of this point as the field detected by a small induction sensor such as a small diameter receiver coil. Figure 4 shows the real and imaginary component of $H_{x}^{s}$ at a single wavenumber with respect to a line at $\mathrm{y}=2 \mathrm{~mm}, \mathrm{z}=2 \mathrm{~mm}$ and along the negative $\mathrm{x}$-axis. The wavenumber is equivalent to aluminium objects $\left(\sigma=3.5 \times 10^{7}\right.$ $\mathrm{S} / \mathrm{m}$ ) subject to a $10 \mathrm{kHz}$ excitation field.

The larger of the four objects, the ellipsoid and cylinder, show good similarity between the finite element and boundary element methods at high wavenumbers. The real components are particularly well matched: Figure 2 shows good correspondence in the vector directions for the two objects and figure 3 shows the boundary element computed real component to be within $20 \%$ of the finite element method at wavenumbers down to $k=10^{3}$. In practical terms, this is equivalent to an aluminium cylinder or ellipsoid at $3.5 \mathrm{kHz}$, or a skin-depth of approximately $1.4 \mathrm{~mm}$. Both objects have a $10 \mathrm{~mm}$ radius (at the widest point tangential to the $\mathrm{x}$-axis for the ellipsoid), which implies the skin-depth should be around $10-15 \%$ of the size of the characteristic dimension of the shape. This broadly agrees with the original work of Sun et al. [7] which found the thin skin approximation applies well when the skin depth is no

\footnotetext{
${ }^{1}$ Animations available at

www.eee.manchester.ac.uk/people/staff/profile/?ea=michael.otoole
} 
greater than an order of magnitude less than the characteristic radius.

The imaginary components show poorer agreement with the finite element results. The boundary element results appear to be approximately double the finite element results at $k=$ $10^{3}$. This result is broadly similar to the findings of Sun et al. [7], where poor match was found with other solvers for the imaginary component when the object material has low permeability. The reason for such poor agreement, given the relatively good agreement of the real component, may be due to poor conformity with the thin skin assumption when the correction factor $\alpha_{n}=1$. Evidently the corners of the cylinder and the ellipsoid surface challenge the assumption of uniform exponential decay of the normal component of the magnetic field. The method may benefit from a rational approach to choosing $\alpha_{n}$. For example, Sun et al. [7] approximates $\alpha_{n}$ using known solutions of a sphere.

The agreement between the boundary and finite element methods for both the real and imaginary components do improve with increasing frequency and conductivity. The asymptotic perfect electrical conductor result for example, that is when the real component approaches $k \rightarrow \infty$, appears to converge very closely.

Figure 4 shows a similar result to figure 3 . The differences between the boundary and finite element is relatively large close to the boundary of the shape. This quickly converges with distance from the base of the shape. Again, the imaginary component shows considerably poorer agreement than the real component, implying limitation of the thin-skin approximation. Figure 4 shows a similar result to figure 3 . The differences between the boundary and finite element is relatively large close to the boundary of the shape. This quickly converges with distance from the base of the shape. Again, the imaginary component shows considerably poorer agreement than the real component, implying limitation of the thin-skin approximation.

The trends found for larger objects also occur for the smaller right-angle and c-shape objects. We find the agreement between boundary and finite element to improve with increasing wavenumber and distance from the boundary. However, the smaller objects show considerably poorer results generally than the larger objects. Here, the ratio between the characteristic dimension to skin-depth is much smaller and the presence of complex geometry further limits the validity of the thinskin approximation. The results for the right angle are only valid at a around $k=10^{4}$; an order of magnitude different from the larger shapes. The paraview visualizations clearly show implausible field lines at $10 \mathrm{kHz}$ but which converge to realistic field lines matching the finite element results at 1 $\mathrm{MHz}$. Interestingly the $\mathrm{C}$-shape PEC result appears to diverge markedly from the finite element results in contrast to the other shapes. This can not be due to the thin-skin assumption as the skin-depth is infinitesimally small for the perfect electrical conductor case.

\section{CONCLUSiON}

In this paper, we evaluated the thin-skin approximation boundary element method for solving electromagnetic scattering problems against an existing, well-established commercial finite element solver. This method uses scalar magnetic potentials and an assumption that the component of the magnetic field normal to the surface of the object decays uniformly, with respect to the normal distance from the surface, across the entire surface of the object. This combination allows for a very compact formulation and keeps the size of the matrix inversion required to solve for the boundary elements relatively small. Thus, the method is computationally efficient and potentially can be solved very quickly compared to other electromagnetic solvers.

We find the boundary element method agrees well with the finite element method for the larger objects and the level of agreement improves as frequency or object condcutivity (in the form of the wavenumber). The imaginary components however, agreed less well than the real component. This may be due to variation from the thin-skin assumption due to object geometry and may be improved by better choice of correction factor $\alpha_{n}$. However, choosing $\alpha_{n}$ for any given geometry is an open question.

The agreement for the smaller and more complex-shaped objects was poor. To some extent this is to be expected, as we can no longer necessarily assume a 'thin-skin' for these shapes. The skin depth fills a significant portion of the characteristic dimensions of these pieces, even at higher wavenumbers.

The thin-skin approximation boundary element method has promise as a method for fast solutions to electromagnetic scattering problems. However, we conclude there are significant shortcomings in its practical application in the case of smaller and complex objects. This limits the usefulness in our target applications - for example, it would only be suitable for sorting larger fractions of scrap metal and for measurements towards the higher end of many typical induction based sensors.

\section{ACKNOWLEDGMENTS}

This research was conducted as part of the project Shreddersort: Selective recovery of non-ferrous metal automotive shredder by combined electromagnetic tensor spectroscopy and laser-induced plasma spectroscopy. The project is funded by the European Union's Seventh Framework Programme for research, technological development and demonstration under grant agreement no. 603676 .

\section{REFERENCES}

[1] W. Gibson, The Method of Moments in Electromagnetics. CRC Press, 2007.

[2] M. Lu, Q. Zhao, P. Hu, W. Yin, and A. Peyton, "Prediction of the asymptotical magnetic polarization tensors for cylindrical samples using the boundary element method," in Sensors Applications Symposium (SAS), 2015 IEEE, April 2015, pp. 1-4.

[3] L. A. Marsh, C. Ktistis, A. Jrvi, D. W. Armitage, and A. J. Peyton, "Three-dimensional object location and inversion of the magnetic polarizability tensor at a single frequency using a walk-through metal detector," Measurement Science and Technology, vol. 24, no. 4, p. 045102, 2013. 

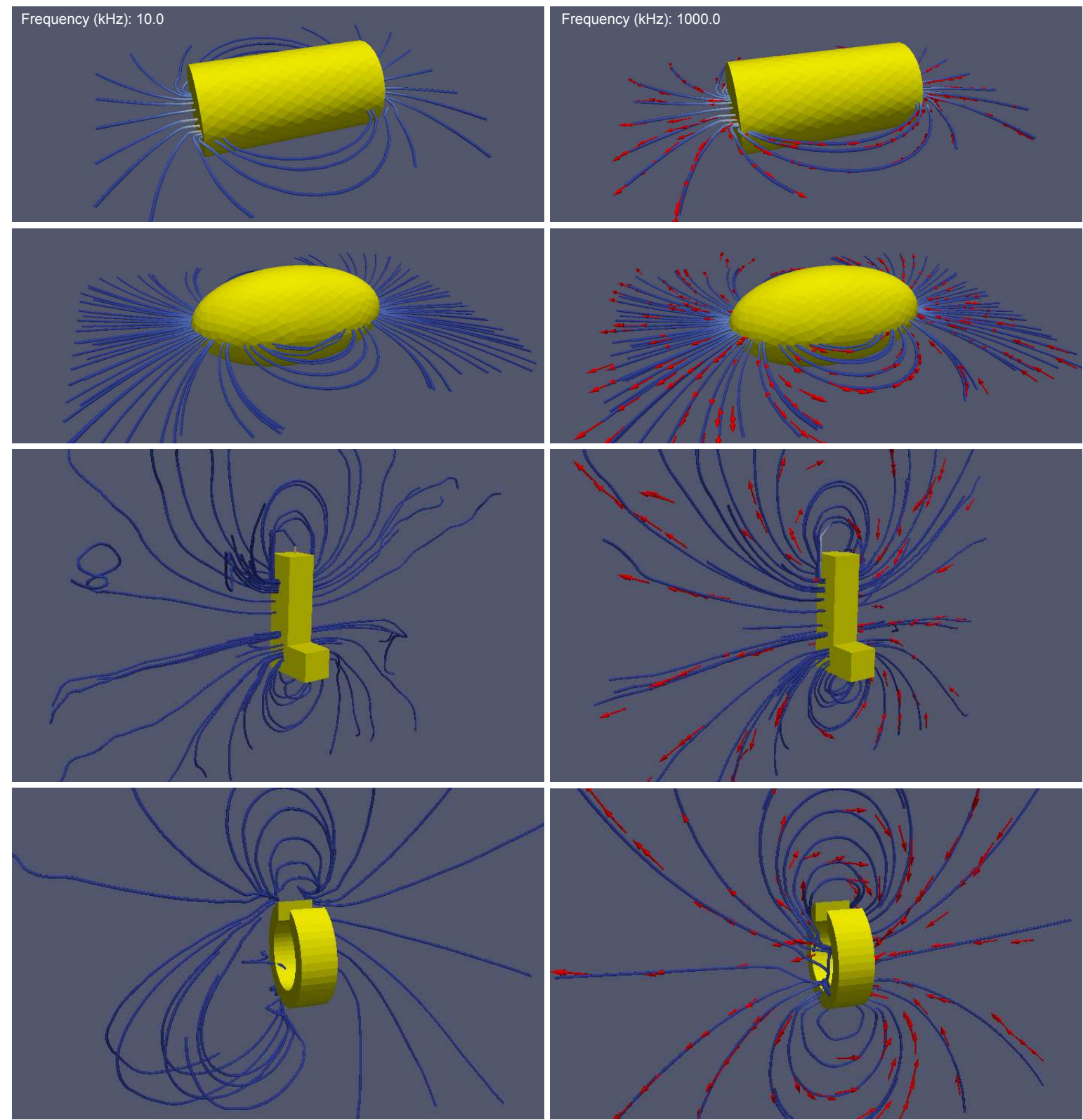

Fig. 2. Example Paraview visualisations of BEM and FEM data. Left-hand side: secondary field BEM data at $10 \mathrm{kHz}$. Right-hand side: BEM data at $1 \mathrm{MHz}$ (streamlines) with the superimposition of the associated FEM data (red glyph arrows).

[4] J. Makkonen, L. A. Marsh, J. Vihonen, A. Jrvi, D. W. Armitage, A. Visa, and A. J. Peyton, "Improving reliability for classification of metallic objects using a wtmd portal," Measurement Science and Technology, vol. 26, no. 10, p. 105103, 2015.

[5] O. Abdel Rehim, J. Davidson, L. Marsh, M. O’Toole, D. Armitage, and A. Peyton, "Measurement system for determining the magnetic polarizability tensor of small metal targets," in Sensors Applications Symposium (SAS), 2015 IEEE, April 2015, pp. 1-5.

[6] J. Makkonen, L. Marsh, J. Vihonen, M. O’Toole, D. Armitage, A. Jarvi, A. Peyton, and A. Visa, "Determination of material and geometric properties of metallic objects using the magnetic polarisability tensor," in Sensors Applications Symposium (SAS), 2015 IEEE, April 2015, pp. $1-5$.

[7] K. Sun, K. O’Neill, F. Shubitidze, S. Haider, and K. Paulsen, "Simulation of electromagnetic induction scattering from targets with negligible to moderate penetration by primary fields," Geoscience and Remote Sensing, IEEE Transactions on, vol. 40, no. 4, pp. 910-927, Apr 2002.
[8] K. Sun, K. O'Neill, F. Shubitidze, I. Shamatava, and K. Paulsen, "Theoretical analysis and range of validity of tsa formulation for application to uxo discrimination," Geoscience and Remote Sensing, IEEE Transactions on, vol. 42, no. 9, pp. 1871-1881, Sept 2004.

[9] J. Schberl, "Netgen an advancing front $2 \mathrm{~d} / 3 \mathrm{~d}$-mesh generator based on abstract rules," Computing and Visualization in Science, vol. 1, no. 1, pp. 41-52, 1997.

[10] G. Rubinacci, A. Tamburrino, S. Ventre, and F. Villone, "Fast computational methods for large-scale eddy-current computation," Magnetics, IEEE Transactions on, vol. 38, no. 2, pp. 529-532, Mar 2002.

[11] R. Graglia, "On the numerical integration of the linear shape functions times the 3-d green's function or its gradient on a plane triangle," Antennas and Propagation, IEEE Transactions on, vol. 41, no. 10, pp. 1448-1455, Oct 1993.

[12] U. Ayachit, The ParaView Guide: A Parallel Visualization Application. USA: Kitware, Inc., 2015. 


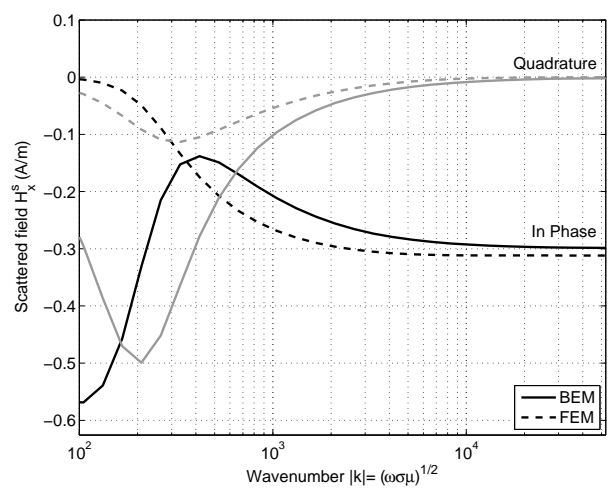

(a)

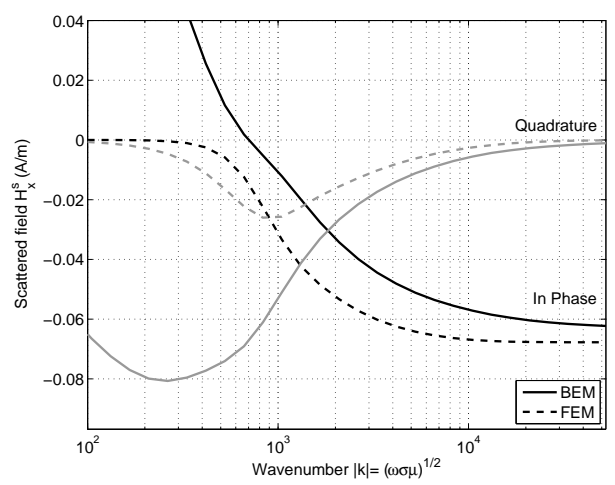

(c)

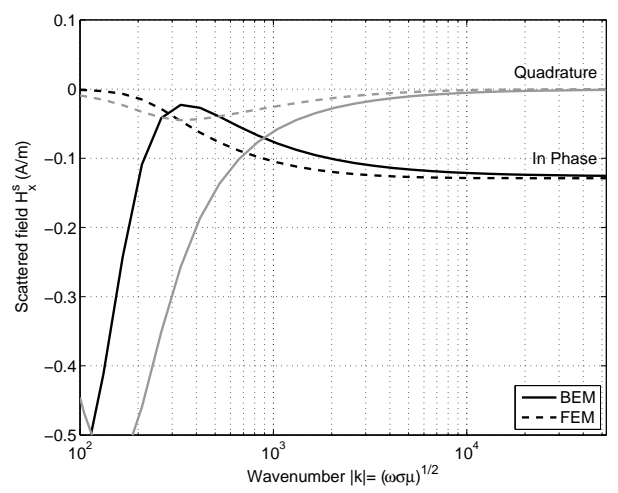

(b)

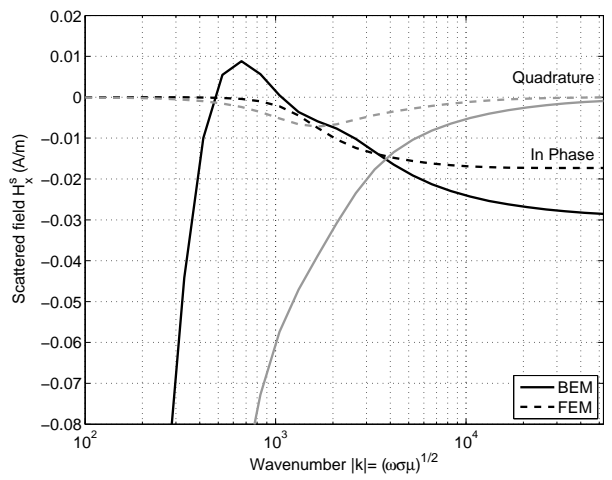

(d)

Fig. 3. The x-axis component of the scattered field $H_{x}^{s}$ at a point $8 \mathrm{~mm}$ below the bottom surface of the object $(\mathrm{y}=2 \mathrm{~mm}, \mathrm{z}=2 \mathrm{~mm}) \mathrm{Vs}$ wavenumber $\mathrm{k}$ $\left(\mathrm{m}^{-1}\right)$.

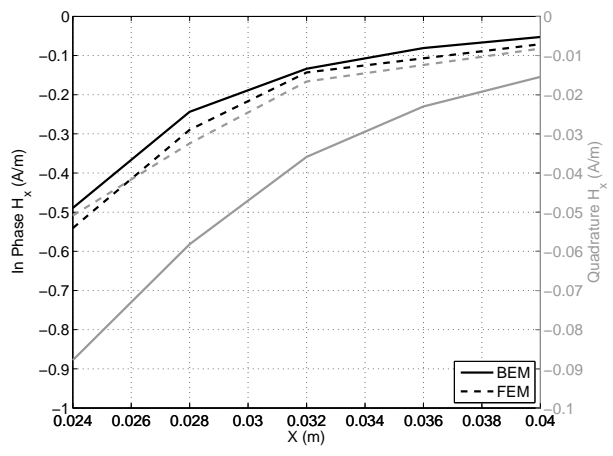

(a)

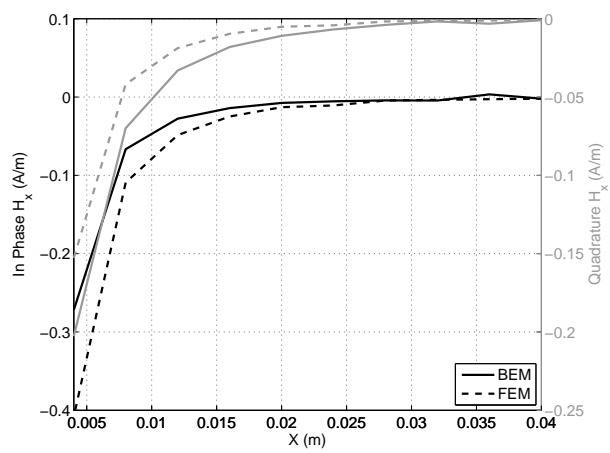

(c)

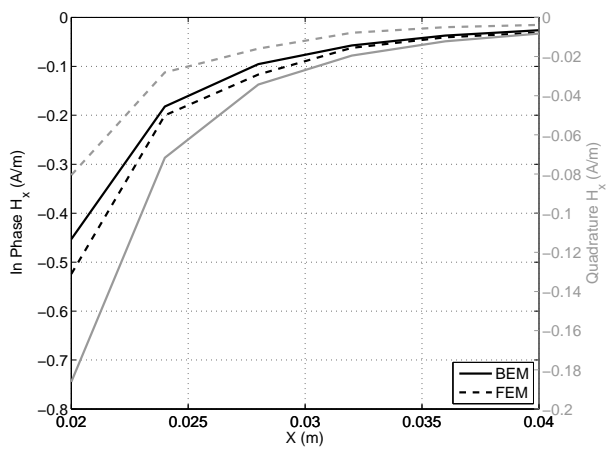

(b)

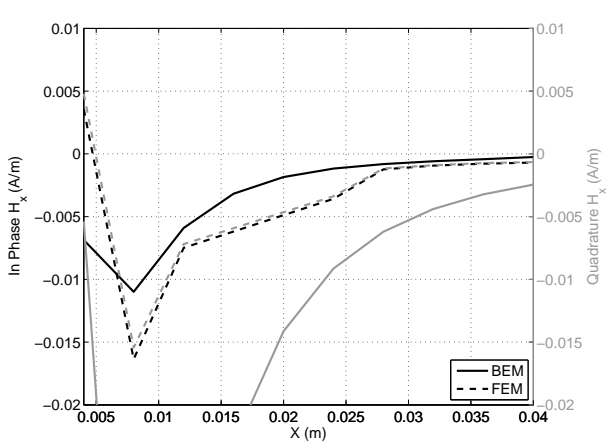

(d)

Fig. 4. The $\mathrm{x}$-axis component of the scattered field $H_{x}^{s}$ on a line $\mathrm{y}=2 \mathrm{~mm}, \mathrm{z}=2 \mathrm{~mm}, \mathrm{x}<0$, projecting from the bottom surface of the object. 